\title{
Applying Geochemistry to Improve Health and Fight Environmental Injustice - Earl Ingerson Lecture
}

\section{GABRIEL FILIPPELLI}

Indiana University Purdue University Indianapolis

Presenting Author: gfilippe@iupui.edu

Global society was harshly awoken to the grim realities of environmental injustice in 2020. First came an environmentally sparked pandemic that strikes those exposed to environmental contaminants particularly hard. Then came the protests again police brutality that included protests against the environmental conditions of many poor communities of color. The connecting lines for the events were the environment, race and class. And, believe it or not, geochemists have tools at their disposal that can become weapons to fight environmental injustice and improve the health and well-being of communities.

One critical tool is the geochemist's unique skillset to constrain and model the cycling of chemical components in the air, water, and soil. Another tool is the ability to apportion fluxes to calculate those components of the cycle that are most important, and on what timescale that flux is active. This perspective has proven critical in my own work, which has focused on lead pollution, exposure and mitigation. Areas that geochemists are not typically trained in include some key topics that can help them bridge the elemental with the personal-e.g., from the presence of lead in the environment, to the net exposure from that lead to a vulnerable individual, and finally towards key ways to mitigate that lead exposure pathway and improve personal or community health. This presentation will highlight one investigator's journey from marine sedimentary geochemist to environmental health expert, in an attempt to provide a more general roadmap to geochemists who are interesting in applying their expertise to improve public health and to fight environmental injustice. 